\title{
Realization of the Finite Mass Method
}

\author{
Peter Leinen \\ Mathematisches Institut, Universität Tübingen \\ leinen@na. uni-tuebingen.de, \\ http://na.uni-tuebingen.de/ peter
}

\begin{abstract}
The finite mass method, a new Lagrangian method for the numerical simulation of gas flow, is presented. The finite mass method is founded on a discretization of mass, not of space. Mass is subdivided into small mass packets. These mass packets move under the influence of internal and external forces. The right-hand sides of the differential equations governing the motion of the particles are integrals which cannot be evaluated exactly. A Lagrangian discretization of these integrals will be presented that maintains the invariance and conservation properties of the method. An efficient way to implement and parallelize the method is discussed.
\end{abstract}

\section{Introduction}

The finite mass method is a new approach to solve the equation of gas dynamics. Contrary to the usual finite volume or finite element methods, the finite mass method is based on a discretization of mass, not of space. Mass is subdivided into small mass packets of finite extension each of which is equipped with finitely many internal degrees of freedom. These mass packets move under the influence of internal and external forces and the laws of thermodynamics and can undergo arbitrary linear deformations.

Numerical methods based on the Lagrangian view of fluid flow like the finite mass method are well-suited for free flows in unbounded space as in astrophysical problems. One of the most popular methods of this type, Monaghan's smoothed particle hydrodynamics (SPH) [4], come from this research area.

The finite mass method is based on the particle model of compressible fluids which has been developed by Yserentant ([5-7]) and is generalized in [1].

Particles do not directly interact by each other but only by global quantities like the mass density. This property is reflected in the data structure. A efficient implementation of the method both on serial and parallel computer architectures is based on a careful analysis of the data flow.

The rest of the paper is organized as follows. In Section 2 the finite mass method is described briefly. It is shown how the particles are built and the mass density is discretized. Derivation of the equation of motion utilizing a Lagrangefunction is done in Section 3. In Section 4 we discuss data structures and the data flow in a realization of the method. A parallel implementation for shared and distributed memory architectures is presented.

P.M.A. Sloot et al. (Eds.): ICCS 2002, LNCS 2330, pp. 470-479, 2002.

(C) Springer-Verlag Berlin Heidelberg 2002 


\section{Mass Packets}

\section{$2.1 \quad$ Local quantities}

The internal mass distribution of a mass packet is described by a continuously differentiable shape function $\psi: \mathbb{R}^{d} \rightarrow \mathbb{R}_{+}, d$ the space dimension, with compact support. We assume that

$$
\int \psi(\mathbf{y}) \mathrm{d} \mathbf{y}=1, \quad \int \psi(\mathbf{y}) \mathbf{y} \mathrm{d} \mathbf{y}=\mathbf{0}, \quad \int \psi(\mathbf{y}) y_{k} y_{l} \mathrm{~d} \mathbf{y}=J \delta_{k l},
$$

with the $y_{k}$ the components of $\mathbf{y}$. The second condition states that the origin of the body coordinates is the center of mass and the third one normalizes the deformation. A suitable choice for the shape function $\psi$ is, for example, the tensor product

$$
\psi(\mathbf{y})=\prod_{k=1}^{d} \widetilde{\psi}\left(y_{k}\right),
$$

of the normalized third order B-spline given by

$$
\widetilde{\psi}(\xi)=\frac{4}{3}\left\{\begin{array}{lrl}
1-6 \xi^{2}(1-|\xi|), & 0 \leq|\xi| \leq 1 / 2 \\
2(1-|\xi|)^{3} & , & 1 / 2 \leq|\xi| \leq 1 \\
0 \quad & 1 \leq|\xi|
\end{array} .\right.
$$

We allow for linear deformation of the particles. That is the points $\mathbf{y}$ of the particle $i$ move along the trajectories

$$
t \rightarrow \mathbf{q}_{i}(t)+\mathbf{H}_{i}(t) \mathbf{y}, \quad \operatorname{det} \mathbf{H}_{i}(t)>0 .
$$

The vector $\mathbf{q}_{i}(t)$ is the position of the particle and the matrix $\mathbf{H}_{i}(t)$ determines the linear deformation of the given particle. For a given point $\mathbf{x}$ in space coordinates

$$
\mathbf{y}=\mathbf{H}_{i}(t)^{-1}\left(\mathbf{x}-\mathbf{q}_{i}(t)\right)
$$

are the body coordinates at time $t$.
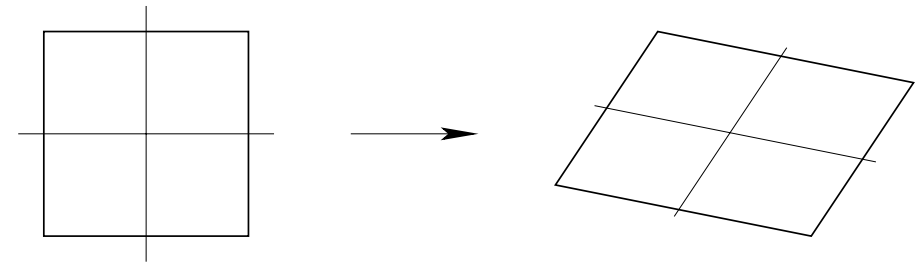

Fig. 1. Transformation of a particle by $\mathbf{q}_{i}(t)+\mathbf{H}_{i}(t) \mathbf{y}$

The points $\mathbf{y}$ of the particle $i$ move with the velocity

$$
t \rightarrow \mathbf{q}_{i}^{\prime}(t)+\mathbf{H}_{i}^{\prime}(t) \mathbf{y} .
$$


With (5) we obtain the velocity field

$$
\mathbf{v}_{i}(\mathbf{x}, t)=\mathbf{q}_{i}^{\prime}(t)+\mathbf{H}_{i}^{\prime}(t) \mathbf{H}_{i}(t)^{-1}\left(\mathbf{x}-\mathbf{q}_{i}(t)\right)
$$

of the particle $i$ in space coordinates. The masses of the particles can be distinct and are denoted by $m_{i}>0$. Of course the $m_{i}$ are always positive.

\subsection{Global quantities}

The superposition of the mass density of the single particles

$$
\rho(\mathbf{x}, t)=\sum_{i=1}^{N} m_{i}\left[\operatorname{det} \mathbf{H}_{i}(t)\right]^{-1} \psi\left(\mathbf{H}_{i}(t)^{-1}\left(\mathbf{x}-\mathbf{q}_{i}(t)\right)\right)
$$

is the density of the total mass. With the abbreviation

$$
\psi_{i}(\mathbf{x}, t)=\left[\operatorname{det} \mathbf{H}_{i}(t)\right]^{-1} \psi\left(\mathbf{H}_{i}(t)^{-1}\left(\mathbf{x}-\mathbf{q}_{i}(t)\right)\right)
$$

this reads as

$$
\rho(\mathbf{x}, t)=\sum_{i=1}^{N} m_{i} \psi_{i}(\mathbf{x}, t) .
$$

In a similar way we get the total mass flux density by

$$
\mathbf{j}(\mathbf{x}, t)=\sum_{i=1}^{N} m_{i} \psi_{i}(\mathbf{x}, t) \mathbf{v}_{i}(\mathbf{x}, t) .
$$

The total velocity field $\mathbf{v}$ is defined by the relation

$$
\mathbf{j}(\mathbf{x}, t)=\rho(\mathbf{x}, t) \mathbf{v}(\mathbf{x}, t)
$$

Beside the mass density we need a second thermodynamic quantity like temperature or entropy to describe the state of a compressible fluid completely. As this is not important for the implementation of the finite mass method we will focus on internal energy $\varepsilon$, which is only a function of the mass density. Ideal gases with

$$
\varepsilon(\rho) \sim\left(\frac{\rho}{\rho_{0}}\right)^{\gamma}
$$

represent the most simple example. For air we have $\gamma=1.4$

\subsection{Equation of motion}

The total internal energy of the system is

$$
V=\int \varepsilon(\rho(\mathbf{x}, t)) \mathrm{d} \mathbf{x} .
$$


The particle $i$ has the kinetic energy

$$
E_{i}(t)=\frac{1}{2} \int m_{i} \psi_{i}(\mathbf{x}, t)\left|\mathbf{v}_{i}(\mathbf{x}, t)\right|^{2} \mathrm{~d} \mathbf{x}
$$

the total kinetic energy of the system is

$$
E(t)=\sum_{i=1}^{N} E_{i}(t)
$$

The equations of motion

$$
\frac{\mathrm{d}}{\mathrm{d} t} \frac{\partial \mathcal{L}}{\partial \mathbf{q}_{i}^{\prime}}-\frac{\partial \mathcal{L}}{\partial \mathbf{q}_{i}}=\mathbf{0}, \quad \frac{\mathrm{d}}{\mathrm{d} t} \frac{\partial \mathcal{L}}{\partial \mathbf{H}_{i}^{\prime}}-\frac{\partial \mathcal{L}}{\partial \mathbf{H}_{i}}=\mathbf{0}
$$

are derived from the Lagrange-function

$$
\mathcal{L}=E-V
$$

which is a function of $\mathbf{q}_{i}, \mathbf{H}_{i}, \mathbf{q}_{i}^{\prime}$ and $\mathbf{H}_{i}^{\prime}$. With the normalized forces

$$
\mathbf{F}_{i}=-\frac{1}{m_{i}} \frac{\partial V}{\partial \mathbf{q}_{i}}, \quad \mathbf{M}_{i}=-\frac{1}{m_{i}} \frac{\partial V}{\partial \mathbf{H}_{i}}
$$

the system of ordinary differential equations read

$$
\mathbf{q}_{i}^{\prime \prime}=\mathbf{F}_{i}, \quad \mathbf{H}_{i}^{\prime \prime}=\frac{1}{J} \mathbf{M}_{i}
$$

Additional frictional forces are necessary to model shocks correctly. These forces transfer kinetic energy into heat, see [1] for a detailed discussion. These frictional forces can be implemented in the same framework as the pressure forces.

\section{Discretization of integrals}

The internal energy (14) and therefore the forces are integrals and cannot not be evaluated exactly. Therefore we have to apply a quadrature rule to compute the forces. As the particle model is invariant to translations and rotations, the quadrature rule should preserve these properties. We start from the identity

$$
\begin{aligned}
\int f(x) \rho(x) \mathrm{d} \mathbf{x} & =\sum_{j=1}^{N} m_{j} \int f(\mathbf{x}) \psi_{j}(\mathbf{x}) \mathrm{d} \mathbf{x} \\
& =\sum_{j=1}^{N} m_{j} \int f\left(\mathbf{q}_{j}+\mathbf{H}_{j} \mathbf{y}\right) \psi(\mathbf{y}) \mathrm{d} \mathbf{y} .
\end{aligned}
$$


The integrals on the right-hand side are replaced by a fixed quadrature formula

$$
\int g(\mathbf{y}) \psi(\mathbf{y}) \mathrm{d} \mathbf{y} \rightarrow \sum_{\nu=1}^{n} \omega_{\nu} g\left(\mathbf{a}_{\nu}\right)
$$

with weights $\omega_{\nu}>0$ and nodes $\mathbf{a}_{\nu}$ inside the support of the shape function $\psi$. The overall result is a quadrature rule

$$
\int f \rho \mathrm{d} \mathbf{x} \rightarrow \sum_{j=1}^{N} m_{j}\left[\sum_{\nu=1}^{n} \omega_{\nu} f\left(\mathbf{q}_{j}+\mathbf{H}_{j} \mathbf{a}_{\nu}\right)\right]=: \int f \mathrm{~d} \mu
$$

with weights $m_{j} \omega_{\nu}$ and nodes $\mathbf{q}_{j}+\mathbf{H}_{j} \mathbf{a}_{\nu}$. The quadrature rule is based on the given discretization of the mass density and not on a subdivision of space into cells. Figure 2 shows the quadrature rule on the reference configuration and the quadrature points generated by two particles.

$$
\begin{array}{|lll|}
\hline+ & + & + \\
+ & + & + \\
+ & + & + \\
\hline
\end{array}
$$

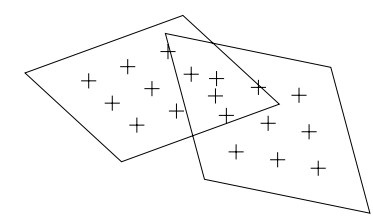

Fig. 2. Quadrature rule

Using the quadrature rule (24) the potential energy (14) is replaced by the fully discrete version

$$
V=\int \tilde{\varepsilon}(\rho) \mathrm{d} \mu,
$$

with $\tilde{\varepsilon}=\varepsilon / \rho$. As both $\tilde{\varepsilon}$ and the quadrature points depend on the $\mathbf{q}_{i}$ and $\mathbf{H}_{i}$, the forces split into two parts

$$
\mathbf{F}_{i}=\mathbf{F}_{i}^{(1)}+\mathbf{F}_{i}^{(2)}, \quad \mathbf{M}_{i}=\mathbf{M}_{i}^{(1)}+\mathbf{M}_{i}^{(2)}
$$

of different structure. Into

$$
\begin{aligned}
\mathbf{F}_{i}^{(1)} & =-\int \tilde{\varepsilon}^{\prime}(\rho) \frac{\partial \psi_{i}}{\partial \mathbf{q}_{i}} \mathrm{~d} \mu, \\
\mathbf{M}_{i}^{(1)} & =-\int \tilde{\varepsilon}^{\prime}(\rho) \frac{\partial \psi_{i}}{\partial \mathbf{H}_{i}} \mathrm{~d} \mu
\end{aligned}
$$

all quadrature points $\mathbf{q}_{j}+\mathbf{H}_{j} \mathbf{a}_{\nu}$ contained in the support of the given particle $i$ enter whereas

$$
\begin{aligned}
\mathbf{F}_{i}^{(2)} & =-\sum_{\nu=1}^{n} \omega_{\nu}(\nabla \tilde{\varepsilon})\left(\mathbf{q}_{i}+\mathbf{H}_{i} \mathbf{a}_{\nu}\right) \\
\mathbf{M}_{i}^{(2)} & =-\sum_{\nu=1}^{n} \omega_{\nu}\left[(\nabla \tilde{\varepsilon})\left(\mathbf{q}_{i}+\mathbf{H}_{i} \mathbf{a}_{\nu}\right)\right]\left[\mathbf{a}_{\nu}\right]^{\mathrm{T}}
\end{aligned}
$$


depend only on values at the quadrature points $\mathbf{q}_{i}+\mathbf{H}_{i} \mathbf{a}_{\nu}$ assigned to the particle $i$ itself.

These kind of discretization of the internal energy guarantees conservation of energy, momentum, and angular momentum. See [1] for a proof of this property.

\section{Realization}

The key observation is that the particles do not directly interact by each other but only by global fields like the mass density, for example. This is reflected in our choice of the data structures. We clearly separate the quadrature points in which global fields are stored from the primary particle information.

\subsection{Data structures}

Our first data structure are the particles. Storage is provided for the masses $m_{i}$, the values $\mathbf{q}_{i}, \mathbf{H}_{i}, \mathbf{q}_{i}^{\prime}$ and $\mathbf{H}_{i}^{\prime}$ and the forces $\mathbf{F}_{i}$ and $\mathbf{M}_{i}$. The second data structure are the quadrature points. A quadrature point contains information on its position $\mathbf{x}_{Q}=\mathbf{q}_{i}+\mathbf{H}_{i} \mathbf{a}_{\nu}$, the associated weights $\omega_{Q}=m_{i} \omega_{\nu}$ and of all relevant field quantities like the mass density $\rho_{Q}=\rho\left(\mathbf{x}_{Q}\right)$ and $\tilde{\varepsilon}_{Q}^{\prime}=\tilde{\varepsilon}^{\prime}\left(\rho_{Q}\right)$.

\subsection{Algorithm}

The computation of the forces $\mathbf{F}_{i}^{(1)}$ and $\mathbf{M}_{i}^{(1)}$ for a given distribution of the particles splits into four steps.

In an initializing step the position $\mathbf{x}_{Q}=\mathbf{q}_{i}+\mathbf{H}_{i} \mathbf{a}_{\nu}$ of the quadrature points. and the weights $\omega_{Q}=m_{i} \omega_{\nu}$ are generated. In this step information is transferred from each particle to the quadrature points associated with this particle.

The next step is to compute the values $\rho_{Q}$ of the mass density at all quadrature points. Information is transferred from a particle $i$ to all quadrature points in the support of $\psi_{i}$. This step is realized as a loop over all particles.

In the third step the values $\tilde{\varepsilon}^{\prime}=\tilde{\varepsilon}^{\prime}\left(\rho_{Q}\right)$ are computed in a loop over all quadrature points. By this a multiple evaluation of $\tilde{\varepsilon}_{Q}^{\prime}$ is avoided.

In the fourth step the forces $\mathbf{F}_{i}^{(1)}$ and $\mathbf{M}_{i}^{(1)}$ are finally computed using the results from the steps above. This step is again realized as a loop over all particles. Information is transferred from the quadrature points in the support of $\psi_{i}$ back to the particle $i$. Table 1 summarize the four steps.

\subsection{Search tree}

Steps 2 and 4 crucially depend on an efficient algorithm to find all quadrature points in the support of a given particle. We use adaptive search trees for this purpose.

The root of the tree is a rectangular box containing all quadrature points. A leaf of the tree is further refined if it contains too many quadrature points. 


\begin{tabular}{|l|c|c|}
\hline step & operation & data flow \\
\hline \hline 1 & $\forall$ particles $i$ & $Q(P) \leftarrow P$ \\
& $\forall$ quadrature points $Q$ of particle $i$ & \\
& $\mathbf{x}_{Q} \leftarrow \mathbf{q}_{i}+\mathbf{H}_{i} \mathbf{a}_{\nu}$ & \\
\hline 2 & $\forall$ particles $i$ & \\
& $\forall$ quadrature points $Q$ at which $\psi_{i}>0$ & \\
& $\rho_{Q} \leftarrow \rho_{Q}+m_{i} \psi_{i}\left(x_{Q}\right)$ & $Q \leftarrow Q$ \\
\hline 3 & $\forall$ quadrature points & \\
\hline 4 & $\tilde{\varepsilon}_{Q}^{\prime} \leftarrow \tilde{\varepsilon}^{\prime}\left(\rho_{Q}\right)$ & \\
& $\forall$ particles $i$ & \\
& $\forall$ quadrature points at which $\psi_{i}>0$ & \\
& $\mathbf{F}_{i} \leftarrow \mathbf{F}_{i}-\omega_{Q} \tilde{\varepsilon}_{Q}^{\prime} \frac{\partial \psi_{i}}{\partial \mathbf{q}_{i}}$ & \\
& $\mathbf{M}_{i} \leftarrow \mathbf{M}_{i}-\omega_{Q} \tilde{\varepsilon}_{Q}^{\prime} \frac{\partial \psi_{i}}{\partial \mathbf{H}_{i}}$ & \\
\hline
\end{tabular}

Table 1. Computation of the forces

All leaves having a non-empty intersection with a particle establish a superset of the set of quadrature points needed in steps 2 and 4 .

To keep the computing time and the amount of memory to store the trees as small as possible, it is important to reorder the quadrature points such that every node of the tree contains only consecutive points. The address of the first and the last of these points is attached to the nodes. This allows to access the quadrature points contained in the domain represented by the node in a very efficient way. Reordering of the quadrature points also improves the data locality in the above algorithm. The nodes of this tree are sorted by means of a Hilbert-like space-filling curve $[2,3,8]$, which again helps to improve the data locality. Figure 3 shows the different levels of the adaptive search tree for a given distribution of points and the corresponding space-filling curve. We have allowed at most 5 points in one cell.

\section{Parallelization}

For ease of presentation here we restrict ourselves to a machine with only two processors. The generalization to the multiprocessor case will be obvious.

The data flow of the algorithm above is the starting point for its parallelization. For the rest of the section we split the set of the particles into two disjoint subsets P1 and P2. By Q1 and Q2 we denote the sets of quadrature points associated with the particles in $\mathbf{P 1}$ and $\mathbf{P 2}$, respectively. The computation of the forces for this situation is shown in Table 2. The interaction between $\mathbf{P} \mathbf{1}$ and Q2, for example, is due to the fact that there may be quadrature points from the set Q2 in the support of particles from P1. In a parallel implementation we have to pay attention to this kind of interaction. 

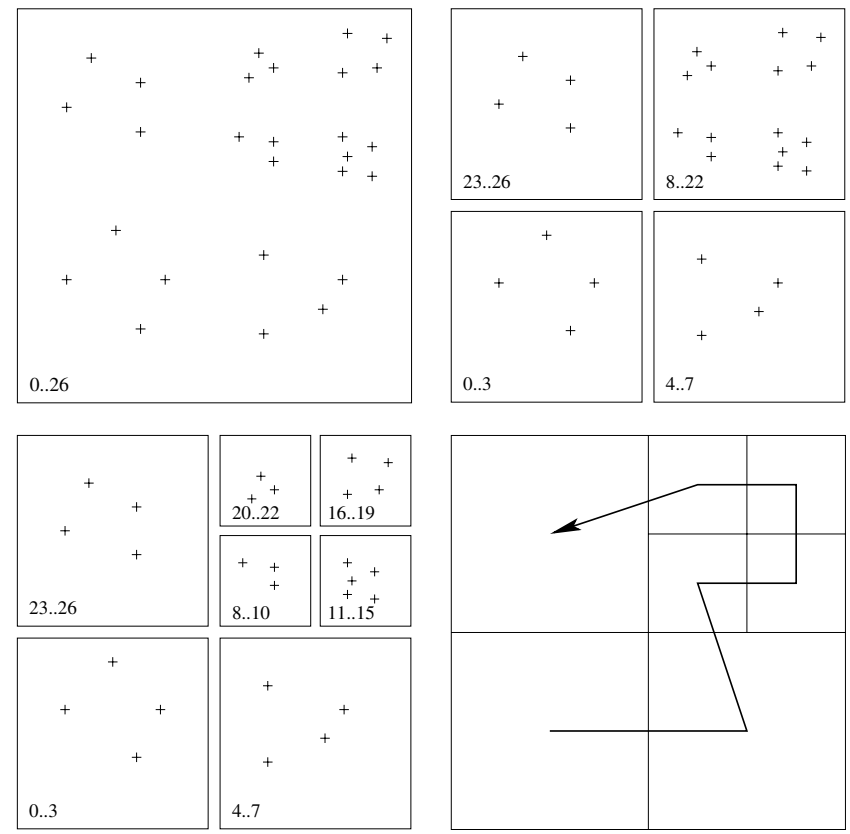

Fig. 3. Adaptive search tree and the space filling curve

\subsection{Shared memory implementation}

First we will discuss how to get a parallel algorithm for a shared memory architecture. The particle sets $\mathbf{P} \mathbf{1}$ and $\mathbf{P 2}$ and correspondingly the sets Q1 and Q2 are now associated with processors 1 and 2. Each processor is allowed to read from all data sets but is only allowed to write to its own data sets. Especially step 2 and step 4 have to be organized in this way. The initialization of the quadrature points as well as the third step are easy to realize in this setting.

The algorithm from Table 2 is organized as shown in Table 3. After the first three steps data have to be synchronized to guarantee that in step 4 the right filed values in the quadrature points are accessed. That is step 4 is not allowed to start before all operations in step 3 are finished.

\subsection{Distributed memory implementation}

The realization on a distributed memory machine is easy when the particle information is redundantly hold on both processors. The realization of the first three steps then does not differ from the shared memory case because in these three steps every processor accesses only data stored in its own memory. No synchronization is needed after step 3. The computation of the forces (step 4) for the distributed memory case is split into a computation and a communication phase. The computational step ends with information in all particles due to one 


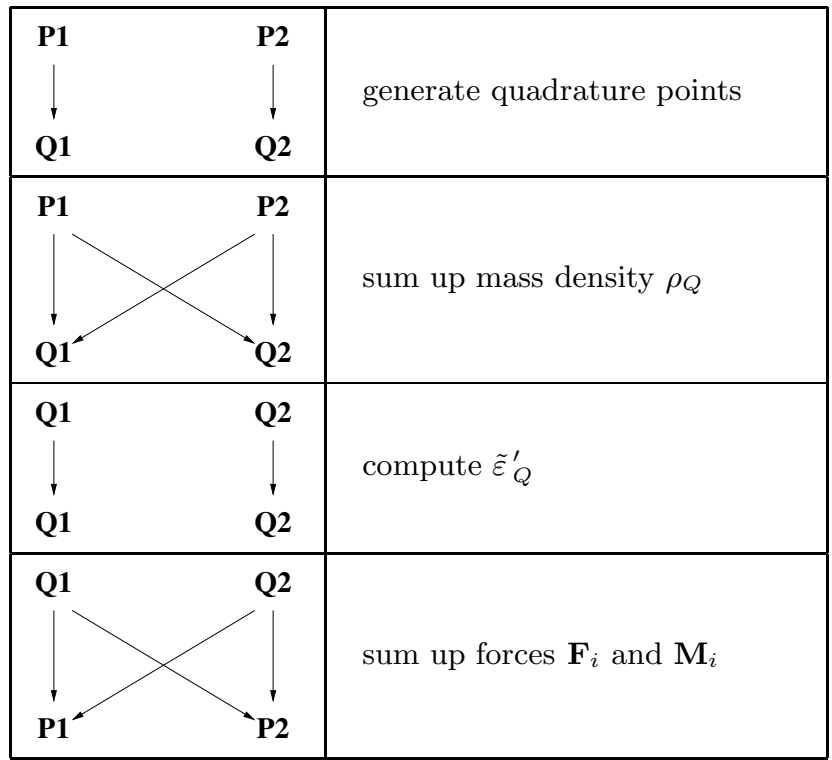

Table 2. Data flow

\begin{tabular}{|c|c|c|c|c|}
\hline \multicolumn{2}{|c|}{ processor 1} & \multirow[b]{2}{*}{ generate quadrature points } & \multicolumn{2}{|c|}{ processor 2} \\
\hline $\begin{array}{c}\text { P1 } \\
\downarrow \\
\text { Q1 }\end{array}$ & $\begin{array}{l}\text { P2 } \\
\text { Q2 }\end{array}$ & & $\begin{array}{l}\text { P1 } \\
\text { Q1 }\end{array}$ & $\begin{array}{c}\mathbf{P 2} \\
\downarrow \\
\mathbf{Q 2}\end{array}$ \\
\hline $\begin{array}{c}\text { P1 } \\
\mid \\
\mathbf{Q 1}^{-}\end{array}$ & Q2 & compute $\rho_{Q}$ & Q1 & Q' \\
\hline $\begin{array}{c}\text { Q1 } \\
\downarrow \\
\text { Q1 }\end{array}$ & $\begin{array}{l}\text { Q2 } \\
\text { Q2 }\end{array}$ & compute $\tilde{\varepsilon}_{Q}^{\prime}$ & $\begin{array}{l}\text { Q1 } \\
\text { Q1 }\end{array}$ & $\begin{array}{c}\text { Q2 } \\
\downarrow \\
\text { Q2 }\end{array}$ \\
\hline \multicolumn{5}{|c|}{ synchronization } \\
\hline $\begin{array}{l}\text { Q1 } \\
\left.\right|_{\mathbf{P 1}} ^{\prime}\end{array}$ & P2 & compute forces & $\begin{array}{l}\text { Q1 } \\
\text { P1 }\end{array}$ & 'P2 \\
\hline
\end{tabular}

Table 3. Shared memory implementation 
set of quadrature points. We obtain the forces if we sum up these values. In Table 4 the implementation of step 4 for the distributed memory case is shown.

\begin{tabular}{|l|l|r|}
\hline \multicolumn{2}{|c|}{ processor 1 } & processor 2 \\
\hline Q1 & & \\
P1 & compute forces & \\
\hline \multicolumn{2}{|c|}{ communication \& synchronization } \\
\hline
\end{tabular}

Table 4. Distributed memory implementation of step 4

For a moderate number of particles this very simple approach is satisfactory since the amount of data stored for the particles is small compared with the data for the quadrature points. In fact we need only copies of those particles which are in the neighborhood of the particles associated with the processor. How to distribute the particles on the processors in a clever and cheap way and how to get information on the copies which are needed, will be discussed in a forthcoming paper.

\section{References}

1. Gauger, Chr., Leinen, P., Yserentant, H.: The finite mass method. SIAM J. Numer. Anal., 37 (2000), 1768-1799

2. Hilbert, D.: Über die stetige Abbildung einer Linie auf ein Flächenstück. Math. Annalen, 38 (1891), 459-460.

3. Sagan, H.: Space-filling curves. Springer, 1994

4. Monaghan, J.J.: Smoothed particle hydrodynamics. Ann. Rev. Astron. Astrophys., 30 (1992), 543-574.

5. Yserentant, H.: A particle model of compressible fluids. Numer. Math., 76 (1997), 111-142.

6. Yserentant, H.: Particles of variable size. Numer. Math., 82 (1999), 143-159.

7. Yserentant, H.: Entropy generation and shock resolution in the particle model of compressible fluids. Numer. Math., 82 (1999), 161-177.

8. Zumbusch, G.: On the quality of space-filling curve induced partitions. Z. Angew. Math. Mech., 81, (2001), 25-28 